\title{
Some Aspects of Control Systems as Dynamical Systems ${ }^{1}$
}

\section{Fritz Colonius ${ }^{2}$ and Wolfgang Kliemann ${ }^{3}$}

Consider the smooth control system

$$
\dot{x}=X_{0}(x)+\sum_{i=1}^{m} u_{i} X_{i}(x)
$$

on a manifold $M$ with admissible controls $u \in \mathscr{U}=\{u: \mathbb{R} \rightarrow U$, locally integrable $\}$ and compact control space $U \subset \mathbb{R}^{m}$. Associated with $(C)$ is a dynamical system

$$
\phi: \mathbb{R} \times \mathscr{U} \times M \rightarrow \mathscr{U} \times M, \quad \phi(t, u, x)=\left(\theta_{t} u, \varphi(t, x, u)\right)
$$

where $\theta_{t}$ is the shift by $t \in \mathbb{R}$ to the right on $\mathscr{U}$, and $\varphi(t, x, u)$ is the solution of (C) at time $t \in \mathbb{R}$ with initial condition $\varphi(0, x, u)=x$, under the control action of $u \in \mathscr{U}$. We discuss some connections between control properties of (C) and basic notions for dynamical systems, such as topological mixing, chain recurrence, recurrence, invariant (ergodic) measures, and their support. It turns out that these concepts for $(D)$ are related to the control sets and chain control sets of (C): A set $D \subset M$ is a control set of (C) iff the lift $\mathscr{D}=\mathrm{cl}\{(u, x) \in \mathscr{U} \times M$, $\varphi(t, x, u) \in D$ for all $t \in \mathbb{B}\}$ to $\mathscr{U} \times M$ is a maximal topologically mixing (transitive) component of $\phi$, similarly for the lifts of chain control sets and the com. ponents of the chain recurrent set of $\phi$. Furthermore, if $\mu$ is an ergodic, invariant measure of $\phi$, then $\pi_{M}$ (supp $\left.\mu\right) \subset D$ for some control set $D \subset M$, and the points $x \in M$ that are contained in control sets, are the projections onto $M$ of $\phi$-recurrent points.

KEY WORDS: Control systems; dynamical systems; control sets; topological mixing; chain recurrence; recurrence; invariant measures.

AMS(MOS) Subject Classifications: $93 \mathrm{C} 10,93 \mathrm{B05}, 58 \mathrm{~F} 11$.

\footnotetext{
${ }^{1}$ Supported in part by NSF Grant DMS-8813976 and DFG Grant Co 124/6-1.

${ }^{2}$ Institut für Mathematik, Universität Augsburg, D 86159 Augsburg, Germany.

${ }^{3}$ Department of Mathematics, Iowa State University, Ames, Iowa 50011.
} 


\section{INTRODUCTION}

Control systems are projections of certain dynamical systems: Let $\mathscr{U}$ denote the space of admissible control functions and $\theta_{t}$ the usual shift to the right by $t \in \mathbb{R}$. Consider the dynamical system

$$
\phi: \mathbb{R} \times \mathscr{U} \times M \rightarrow \mathscr{U} \times M, \quad \phi(t, u, x)=\left(\theta_{t} u, \varphi(t, x, u)\right)
$$

where $\varphi(t, x, u)$ is the solution of the control equation

$$
\dot{x}(t)=X_{0}(x(t))+\sum_{i=1}^{m} u_{i}(t) X_{i}(x(t))
$$

at time $t \in \mathbb{R}$ under the control $u \in \mathscr{U}$, with initial value $\varphi(0, x, u)=x \in M$, a smooth manifold $M$.

If $\phi(\cdot, u, x)=\left\{\left(\theta_{t} u, \varphi(t, x, u)\right), t \in \mathbb{R}\right\}$ denotes an orbit of (1.1), then the orbits of the control system (1.2) are given by

$$
\begin{aligned}
\mathcal{O}(x) & =\{y \in M, \text { there exists } u \in \mathscr{U} \text { and } t \in \mathbb{R} \text { with } \varphi(t, x, u)=y\} \\
& =\bigcup_{\mathcal{u} \in \mathscr{U}} \pi_{M}(\phi(\cdot, u, x))
\end{aligned}
$$

where $\pi_{M}: \mathscr{U} \times M \rightarrow M$ is the projection onto the second component. Likewise reachable and controllable sets of (1.2) are defined as projections of the positive orbits $\phi(t \geqslant 0, u, x)$, and the negative orbits $\phi(t \leqslant 0, u, x)$ respectively. Therefore certain concepts and properties of the dynamical system (1.1) can be interpreted in terms of the control system (1.2). Vice versa, if $D \subset M$ is a control set of (1.2), i.e., a set where approximate controllability holds (cf. Definition 3.1 below), then $D$ can be lifted to $\mathscr{U} \times M$ as

$$
\mathscr{D}=\operatorname{cl}\{(u, x) \in \mathscr{U} \times M, \varphi(t, x, u) \in \text { int } D \text { for all } t \in \mathbb{R}\}
$$

and $\mathscr{D}$ can be characterized in terms of the theory of dynamical systems. This applies in particular in the trivial case, where (1.2) is completely controllable on $M$.

In this paper we are interested in the transfer mechanism between the dynamical system (1.1) and the control system (1.2) with respect to concepts that are related to recurrence, such as topological mixing, existence and support of invariant measures, chain recurrence, and recurrence. As a by-product we will show that control systems are chaotic [in the sense of Devaney (13)] on their control sets. The results obtained here have various implications for dynamical and control systems: In Ref. 7 we apply them to the theory of linear control semigroups on the projective spaces $\mathbb{P}^{d-1}$, 
$d \geqslant 2$; in that paper we also describe the relation of linear flows on vector bundles to (linearized) control systems. In Ref. 8 the relation between controllability and limit sets of trajectories as time tends to infinity is studied.

The first step in analyzing the dynamical system (1.1) is the definition of an appropriate topology on $\mathscr{U}$, the space of control functions. We will use the weak*-topology on $\mathscr{U} \subset L^{\infty}$, because it implies the uniform convergence on compact time intervals of the corresponding trajectories of (1.2), and therefore it seems appropriate for the flow $\phi$ on $\mathscr{U} \times M$. Kaul (16) associated with a generalized control system [in the sense of Roxin (22)] a semidynamical system $\pi: \mathscr{C} \times \mathbb{R}_{+} \rightarrow \mathscr{C}$, where $\mathscr{C}$ is the trajectory space of a generalized control system. He showed that $\pi$ is continuous, when $\mathscr{C}$ is equipped with the compact open topology.

Section 2 of this paper is devoted to the analysis of the system $(\mathscr{U}, \theta)$ : We show that the periodic points are dense in $\mathscr{U}, \theta$ is continuous, is topologically mixing and transitive, and has sensitive dependence on initial conditions, i.e., $(\mathscr{U}, \theta)$ is chaotic in the sense of Devaney (13). In Section 3 it is proved that $(\mathscr{U} \times M, \phi)$ enjoys the same properties, when restricted to the lifted control sets $\mathscr{D}$, and a characterization of the maximal topologically mixing components in terms of control sets of (1.2) is given.

Section 5 analyzes the invariant measures of $\phi$, their ergodic decomposition and the supports, again with respect to control sets of (1.2). In Section 4 recurrence and chain recurrence of (1.1) are discussed: While the components of the chain recurrent set are reflected in so-called chain control sets of (1.2) on $M$, the situation turns out to be different for recurrent points, because the shift $(\mathscr{U}, \theta)$ is not recurrent.

Hence we obtain the following global picture: Some properties of $(\mathscr{U} \times M, \phi)$ are characterized already through the control theoretic behavior of (1.2) on $M$, e.g., topological mixing is determined via control sets, chain recurrence via chain control sets. Other concepts for (1.1), such as invariant measures and their supports, or recurrence, are described in the $M$-components through control theoretic concepts, i.e., control sets, but now the $\mathscr{U}$-component contains additional information. References 8 and 9 contain further results in these directions.

\section{THE SHIFT SPACE $(\mathscr{U}, \theta)$}

Let $U \subset \mathbb{R}^{m}, m \geqslant 1$, be compact and convex, and denote

$$
\mathscr{U}=\{u: \mathbb{R} \rightarrow U, u \text { locally integrable }\}
$$

Define by $\theta_{t}$ the shift to the right by $t \in \mathbb{R}$, i.e.,

$$
\theta: \mathbb{R} \times \mathscr{U} \rightarrow \mathscr{U}, \quad \theta(t, u(\cdot))=u(\cdot+t)
$$

For $\theta(t, \cdot): \mathscr{U} \rightarrow \mathscr{U}$ we will often use the notation $\theta_{t}$. 
We will first analyze the set $\mathscr{U}$, equipped with the weak*-topology. Note that $\mathscr{U} \subset L^{\infty}\left(\mathbb{R}, \mathbb{R}^{m}\right)$.

2.1. Lemma. The set $\mathscr{U}$ is compact and metrizable in the weak*topology of $L^{\infty}\left(\mathbb{R}, \mathbb{R}^{m}\right)=\left(L^{1}\left(\mathbb{R}, \mathbb{R}^{m}\right)\right)^{*}$, a metric is given by

$$
d(u, v)=\sum_{n=1}^{\infty} \frac{1}{2^{n}} \frac{\left|\int_{\mathbb{R}}\left\langle u(t)-v(t), x_{n}(t)\right\rangle d t\right|}{1+\left|\int_{\mathbb{R}}\left\langle u(t)-v(t), x_{n}(t)\right\rangle d t\right|}
$$

where $\left\{x_{n}, n \in \mathbb{N}\right\}$ is a countable, dense subset of $L^{1}\left(\mathbb{R}, \mathbb{R}^{m}\right)$. With this metric $\mathscr{U}$ is a compact, complete, separable metric space. (Here $\langle\cdot, \cdot\rangle$ denotes an inner product in $\mathbb{R}^{m}$.)

Proof. The dual space of $L^{1}\left(\mathbb{R}, \mathbb{R}^{m}\right)$ is $L^{\infty}\left(\mathbb{R}, \mathbb{R}^{m}\right)$ (see, e.g., Ref. 5, Theorem 4.5.1, and recall that the Lebesgue measure on $\mathbb{R}$ is $\sigma$-finite). Furthermore, $L^{1}\left(\mathbb{R}, \mathbb{R}^{m}\right)$ is separable (see, e.g., Ref. 5, Proposition 3.4.5, and recall that the $\sigma$-algebra of Borel sets in $\mathbb{R}$ is countably generated). Now the dual space of a separable Banach space has a compact, metrizable unit ball in the weak*-topology, and a metric is given by (2.1) (see, e.g., Ref. 14, Theorem V.5.1). By Alaoglu's theorem $\mathscr{U}$ is weak* compact, hence a compact metric space. Thus $\mathscr{U}$ is complete (see, e.g., Ref. 15, Theorem 4.3.28) and also separable (see, e.g., Ref. 15; Theorem 4.3.5 and 4.3.27).

From now on we will consider $\mathscr{U}$ as a metric space with metric given by (2.1).

2.2. Lemma. The periodic functions are dense in $\mathscr{U}$.

Proof. Pick $u^{0} \in \mathscr{U}$ and let $W$ be a neighborhood of $u^{0}$. Then (cf., e.g., Ref. 23, p. 31) there are $\varepsilon>0$ and $x_{1}, \ldots, x_{k} \in L^{1}\left(\mathbb{R}, \mathbb{R}^{m}\right)$ with

$$
\left\{\begin{array}{c}
u \in L^{\infty}\left(\mathbb{R}, \mathbb{R}^{m}\right) ; u(t) \in U \text { a.e. } \quad \text { and } \\
\left|\int_{\mathbb{R}}\left\langle u^{0}(t)-u(t), x_{j}(t)\right\rangle d t\right|<\varepsilon \text { for } j=1, \ldots, k
\end{array}\right\} \subset W
$$

Since $x_{j} \in L^{1}\left(\mathbb{R}, \mathbb{R}^{m}\right)$, there is $T>0$ such that for $j=1, \ldots, k$

$$
\int_{\mathbb{R} \backslash[-T, T]}\left|x_{j}(t)\right| d t<\varepsilon \frac{1}{\operatorname{diam} U}
$$


where $\operatorname{diam} U:=\sup \left\{\left|u_{1}-u_{2}\right|, u_{1}, u_{2} \in U\right\}<\infty$. Define $u_{p}(t)=u^{0}(t)$ for $t \in[-T, T]$, and extend $u_{p} 2 T$-periodically to $\mathbb{R}$. Then $u_{p} \in \mathscr{U}$ and

$$
\begin{aligned}
\left|\int_{\mathbb{R}}\left\langle u^{0}(t)-u_{p}(t), x_{j}(t)\right\rangle d t\right| & =\left|\int_{\mathbb{R} \backslash[-T, T]}\left\langle u^{0}(t)-u(t), x_{j}(t)\right\rangle d t\right| \\
& \leqslant \operatorname{diam} U \int_{\mathbb{R} \backslash[-T, T]}\left|x_{j}(t)\right| d t \\
& <\varepsilon
\end{aligned}
$$

2.3. Remark. Note that $u \in \mathscr{U}$ is a periodic function iff $u$ is a periodic point of $(\mathscr{U}, \theta)$. Hence Lemma 2.2 says that the periodic points are dense in $(\mathscr{U}, \theta)$.

We now turn to the analysis of the shift space $(\mathscr{U}, \theta)$.

2.4. Lemma. The shift $\theta$ defines a continuous dynamical system on $\mathscr{U}$.

Proof. Obviously $\theta_{t+s}=\theta_{t} \circ \theta_{s}$ and $\theta(0, u)=u$. It remains to prove continuity of $\theta$ : Let $t_{n} \rightarrow t$ in $\mathbb{R}, u_{n} \rightarrow u$ in $\mathscr{U}$. Then for all $x \in L^{1}\left(\mathbb{R}, \mathbb{R}^{m}\right)$

$$
\begin{aligned}
& \left|\int_{\mathbb{R}}\left\langle u_{n}\left(t_{n}+\tau\right), x(\tau)\right\rangle d \tau-\int_{\mathbb{R}}\langle u(t+\tau), x(\tau)\rangle d \tau\right| \\
& \leqslant\left|\int_{\mathbb{R}}\left\langle u_{n}\left(t_{n}+\tau\right)-u_{n}(t+\tau), x(\tau)\right\rangle d \tau\right| \\
& \quad+\left|\int_{\mathbb{R}}\left\langle u_{n}(t+\tau)-u(t+\tau), x(\tau)\right\rangle d \tau\right| \\
& =\left|\int_{\mathbb{R}}\left\langle u_{n}(\tau), x\left(\tau-t_{n}\right)\right\rangle d \tau-\int_{\mathbb{R}}\left\langle u_{n}(\tau), x(\tau-t)\right\rangle d \tau\right| \\
& \quad+\left|\int_{\mathbb{R}}\left\langle u_{n}(\tau)-u(\tau), x(\tau-t)\right\rangle d \tau\right|
\end{aligned}
$$

The second summand converges to zero because $u_{n} \rightarrow u$ in $\mathscr{U}$, the first one can be estimated by

$$
\leqslant \sup _{w \in U}|w| \int_{\mathbb{R}}\left|x\left(\tau-t_{n}\right)-x(\tau-t)\right| d \tau
$$

which converges to zero as $t_{n} \rightarrow t$ (see, e.g., Ref. 21). Hence $\theta\left(t_{n}, u_{n}\right)=$ $u_{n}\left(t_{n}+\cdot\right) \rightarrow u(t+\cdot)$ in $\mathscr{U}$.

In order to describe the properties of the system $(\mathscr{U}, \theta)$, we recall some definitions from topological dynamics (cf., e.g., Ref. 19). 
2.5. Definition. Let $(S, \psi)$ be a continuous time dynamical system on a compact metric space $S$. The limit set $\omega(x)$ of $x \in S$ is defined as

$$
\omega(x)=\left\{y \in S, \text { there exists } t_{k} \rightarrow \infty \text { in } \mathbb{R} \text { with } \psi\left(t_{k}, x\right) \rightarrow y\right\}
$$

$(S, \psi)$ is called topologically transitive, if there exists some $x \in S$ such that $\omega(x)=S$, and topologically mixing, if for any two open sets $V_{1}, V_{2} \subset S$, there exist $T_{0} \in \mathbb{R}, T_{1}>0$ such that for all $n \in \mathbb{N}$,

$$
\psi\left(-n T_{1}+T_{0}, V_{1}\right) \cap V_{2} \neq \varnothing
$$

Note that $\omega(x)$ is closed, compact, and invariant.

2.6. Proposition. The dynamical system $(\mathscr{U}, \theta)$ is topologically mixing and transitive.

Proof. We will show that $\theta$ is topologically mixing. Transitivity then follows in complete analogy to Ref. 19, Proposition I.11.4 and its proof.

It suffices to consider a basis of the weak*-topology on $\mathscr{U}$. Hence we may assume that $V_{1}$ and $V_{2}$ are given by

$$
V_{j}=\left\{v \in \mathscr{U},\left|\int_{\mathbb{B}}\left\langle u_{j}(\tau)-v(\tau), y_{i j}(\tau)\right\rangle d \tau\right|<\varepsilon, i=1, \ldots, k_{j}\right\}
$$

where $u_{j} \in \mathscr{U}, \varepsilon>0, k_{j} \in \mathbb{N}$, and $y_{i j} \in L^{1}\left(\mathbb{R}, \mathbb{R}^{m}\right)$ for $i=1, \ldots, k_{j}, j=1,2$. There exists $T>0$ such that for all $i, j$

$$
\int_{\mathbb{R} \backslash[-T, T]}\left|y_{i j}(\tau)\right| d \tau<\varepsilon \cdot \frac{1}{\operatorname{diam} U}
$$

Let $t>2 T$ be arbitrary and define

$$
v(\tau)= \begin{cases}u_{1}(\tau) & \tau \in(-T, \infty) \\ u_{2}(\tau+t) & \tau \in(-\infty,-T]\end{cases}
$$

Then $v \in V_{1}$, because for $i=1, \ldots, k_{1}$

$$
\begin{aligned}
\left|\int_{\mathbb{R}}\left\langle u_{1}(\tau)-v(\tau), y_{i 1}(\tau)\right\rangle d \tau\right| & \leqslant \int_{-\infty}^{-T}\left|\left\langle u_{1}(\tau)-v(\tau), y_{i 1}(\tau)\right\rangle\right| d \tau \\
& \leqslant \operatorname{diam} U \int_{-\infty}^{-T}\left|y_{i 1}(\tau)\right| d \tau \\
& <\varepsilon
\end{aligned}
$$


On the other hand, we have that $\theta(-t, v) \in V_{2}$, because for $i=1, \ldots, k_{2}$

$$
\begin{aligned}
\left|\int_{\mathbb{R}}\left\langle u_{2}(\tau)-v(\tau-t), y_{i 2}(\tau)\right\rangle d \tau\right| & =\left|\int_{\mathbb{B}}\left\langle u_{2}(\tau+t)-v(\tau), y_{i 2}(\tau+t)\right\rangle d \tau\right| \\
& =\left|\int_{-T}^{\infty}\left\langle u_{2}(\tau+t)-u_{1}(\tau), y_{i 2}(t+\tau)\right\rangle d \tau\right| \\
& =\left|\int_{t-T}^{\infty}\left\langle u_{2}(\tau)-u_{1}(\tau-t), y_{i 2}(\tau)\right\rangle d \tau\right| \\
& \left.\leqslant \operatorname{diam} U \cdot \int_{T}^{\infty}\left|y_{i 2}(\tau)\right| d \tau \text { (since } t-T \geqslant T\right) \\
& <\varepsilon
\end{aligned}
$$

Because $t>2 T$ was arbitrary, this proves the claim.

2.7. Remark. We have shown that the dynamical system $(\mathscr{U}, \theta)$ is topologically mixing and has a dense set of periodic points. Banks et al. (3) prove that these two properties imply sensitive dependence on initial conditions, i.e., there exists $\delta>0$ such that for all $u \in \mathscr{U}$ and each neighborhood $N$ of $U$, there are $v \in N$ and $t>0$ with $d\left(\theta_{i} u, \theta_{t} v\right) \geqslant \delta$. In fact, the proof given there for discrete time systems applies with the obvious modifications to the continuous time case, if one assumes that there are two different periodic orbits. For the shift on $\mathscr{U}$ this can be guaranteed if $U$ contains more that one point.

Thus, in this case, the shift space is chaotic in the sense of Devaney (13), Definition 8.5 .

\section{THE DYNAMICAL SYSTEM $(\mathscr{U} \times M, \phi)$}

The following class of nonlinear control systems will be considered:

$$
\dot{x}(t)=X_{0}(x(t))+\sum_{i=1}^{m} u_{i}(t) X_{i}(x(t))
$$

on a paracompact, Riemannian $C^{\infty}$ manifold $M$ (of dimension $d<\infty$ ), where $X_{0}, \ldots, X_{m}$ are $C^{\infty}$ vector fields on $M$,

$$
u=\left(u_{i}, i=1, \ldots, m\right) \in \mathscr{U}=\{u: \mathbb{R} \rightarrow U \text { locally integrable }\}
$$

and $U \subset \mathbb{R}^{m}$ is compact and convex and contains at least two points. We assume that for all $u \in \mathscr{U}$, all $x \in M$, Eq. (3.1) has a (unique) solution 
$\varphi(t, x, u)$, defined for all $t \in \mathbb{R}$ with $\varphi(0, x, u)=x$. In order to keep some notation simple, we assume that the distribution $\Delta_{\mathscr{L}}$, generated by the Lie algebra $\mathscr{L}=\mathscr{L} \mathscr{A}\left\{X_{0}+\sum u_{i} X_{i},\left(u_{i}\right) \in U\right\}$, is integrable, i.e.,

$$
\operatorname{dim} \Delta_{\mathscr{L}}(x)=d \quad \text { for all } \quad x \in M
$$

The positive orbits of (3.1) at time $t \geqslant 0$ are defined as

$$
\mathcal{O}_{i}^{+}(x)=\{y \in M \text {, there exists } u \in \mathscr{U} \text { such that } y=\varphi(t, x, u)\}
$$

and the reachable set from $x \in M$ using controls in $\mathscr{U}$ is

$$
\mathcal{O}^{+}(x)=\bigcup_{t \geqslant 0} \mathcal{O}_{t}^{+}(x)
$$

Similarly the negative orbit for $t \geqslant 0$ is

$$
\mathcal{O}_{t}^{-}(x)=\{y \in M, \text { there exists } u \in \mathscr{U} \text { such that } x=\varphi(t, y, u)\}
$$

and

$$
\mathcal{O}^{-}(x)=\bigcup_{t \geqslant 0} \mathcal{O}_{t}^{-}(x)
$$

While (H) implies that int $\mathcal{O}^{+}(x) \neq \varnothing$ and int $\mathcal{O}^{-}(x) \neq \varnothing$ for all $x \in M$, this does not mean that system (3.1) is completely controllable on $M$ [i.e., $\left.\mathcal{O}^{+}(x)=M\right]$. Therefore, we define the sets in which (3.1) can actually be controlled (compare Ref. 1).

3.1. Definition. A set $D \subset M$ is called a control set of (3.1), if (i) $D \subset \overline{\mathcal{O}^{+}(x)}$ for all $x \in D$, (ii) for every $x \in D$ there is $u \in \mathscr{U}$ such that the corresponding trajectory of (3.1) satisfies $\varphi(t, x, u) \in D$ for all $t \in \mathbb{R}$, and (iii) $D$ is maximal (w.r.t. set inclusion) with properties (i) and (ii).

3.2. Remark. The definition of control sets given above differs in two points from the one used, e.g., in Refs. 1 and 6. First, measurable controls instead of piecewise constant ones are employed. This does not matter, since only approximate controllability is involved. The second, more important change is the addition of condition (ii) which implies that control sets must be viable in the sense of J. P. Aubin. If assumption $(\mathrm{H})$ holds, every maximal set satisfying (i) and having nonvoid interior also satisfies (ii). This follows from the properties indicated in the following remark.

3.3. Rernark. Under assumption $(H)$ we have exact controllability if int $D \neq \varnothing$, i.e., $\mathcal{O}^{+}(x) \supset \operatorname{int} D$ for all $x \in D$, and $\overline{\operatorname{int} D}=\bar{D}$. Control sets are 
pairwise disjoint and, in general, neither open nor closed. If $M$ is compact, there are at least one closed and one open control set $D$ in $M$. Compare for these results, e.g., Ref. 6.

To the control system (3.1) we associate a control flow in the following way:

$$
\phi: \mathbb{R} \times \mathscr{U} \times M \rightarrow \mathscr{U} \times M, \quad \phi(t, u, x)=(\theta(t, u), \varphi(t, x, u))
$$

where $\theta$ is the shift discussed in Section 2, and $\varphi(t, x, u)$ is the trajectory of (3.1), corresponding to $u$ and $x$. Note that $\phi$ is a flow, because $\phi_{t+s}=\phi_{t} \circ \phi_{s}$, and so is the shift on $\mathscr{U}$ by Lemma 2.4. The $M$-component, however, satisfies $\varphi(t+s, x, u)=\varphi(t, \varphi(s, x, u), \theta(s, u))$, i.e., $\phi$ is a skewproduct flow.

3.4. Lemma. The flow $\phi$ defines a continuous dynamical system on $\mathscr{U} \times M$.

Proof. According to the remarks above, all we have to show is the continuity of $\phi$. But this follows from Lemma 2.4 above and Gronwall's lemma; compare Ref. 6, proof of Lemma 3.4.

Over the control sets of (3.1) the dynamical system (3.2) shares all the properties of the shift space as discussed in Section 2. To be more precise, we lift control sets $D \subset M$ with nonvoid interior to the set $\mathscr{U} \times M$ and define

$$
\mathscr{D}=\operatorname{cl}\{(u, x) \in \mathscr{U} \times M, \varphi(t, x, u) \in \text { int } D \text { for all } t \in \mathbb{R}\}
$$

where the closure is taken with respect to the weak* topology in $\mathscr{U}$ and the given topology on $M$. Observe that $\mathscr{D}$ as well as $\{(u, x) \in \mathscr{U} \times M$, $\varphi(t, x, u) \in$ int $D$ for all $t \in \mathbb{R}\}$ are invariant under $\phi$, and if $D$ is bounded in the metric given by the Riemannian structure on $M$, then $\mathscr{D}$ is compact.

3.5. Proposition. Let $D$ be a control set of (3.1) with int $D \neq \varnothing$, and define $\mathscr{D} \subset \mathscr{U} \times M$ according to (3.3). Then

(i) the periodic points of $\phi$ are dense in $\mathscr{D}$,

(ii) $\phi_{\mid \mathscr{D}}$ is topologically mixing and transitive, and

(iii) $\phi_{\mid \mathscr{D}}$ has sensitive dependence on initial conditions.

Proof. (i) It suffices to show that each $(u, x) \in \mathscr{D}$ with $\varphi(t, x, u) \in$ int $D$ for all $t \in \mathbb{R}$ can be approximated by periodic points. 
Pick $(u, x)$ and let $W=(V \times N) \cap \mathscr{D}, N \subset M, V \subset \mathscr{U}$, be a neighborhood of $(u, x)$. We may assume that $V$ has the form

$$
\left\{v \in \mathscr{U},\left|\int_{\mathbb{R}}\left\langle u(t)-v(t), x_{i}(t)\right\rangle d t\right|<\varepsilon \text { for } i=1, \ldots, k\right\} \text { with } x_{i} \in L^{1}\left(\mathbb{R}, \mathbb{R}^{m}\right)
$$

There exists $T>0$ such that for $i=1, \ldots, k$,

$$
\int_{\mathbb{R} \backslash[-T, T]}\left|x_{i}(t)\right| d t<\frac{\varepsilon}{\operatorname{diam} U}
$$

By assumption on $(u, x), \varphi(-T, x, u)$ and $\varphi(T, x, u) \in$ int $D$, hence, by exact controllability in int $D$, there are $T_{1}>0$ and $u^{0} \in \mathscr{U}$ with

$$
\varphi\left(T_{1}, \varphi(T, x, u), u^{0}\right)=\varphi(-T, x, u)
$$

Define

$$
u_{p}(t)= \begin{cases}u(t) & t \in[-T, T] \\ u^{0}(t-T) & t \in\left(T, T+T_{1}\right)\end{cases}
$$

and extend $u_{p}$ to a $\left(2 T+T_{1}\right)$-periodic function on $\mathbb{R}$. The corresponding trajectory $\varphi(\cdot, x, u)$ is also periodic with period $2 T+T_{1}$ and contained in int $D$ by maximality of control sets. Thus $\left(u_{p}, x\right)$ is a $\phi$-periodic point in $\mathscr{D}$. Furthermore, for $i=1, \ldots, k$,

$$
\left|\int_{\mathbb{R}}\left\langle u(t)-u_{p}(t), x_{i}(t)\right\rangle d t\right| \leqslant \operatorname{diam} U \cdot \int_{\mathbb{R} \backslash[-T, T]}\left|x_{i}(t)\right| d t<\varepsilon
$$

i.e., $u_{p} \in V$ and thus $\left(u_{p}, x\right) \in W$.

Note that this even shows that all points in int $D$ are on some periodic trajectory of $\phi_{\mid \mathscr{D}}$.

(ii) We have to show that for every pair $W_{1}, W_{2}$ of open sets in $\mathscr{D}$, there exist $T_{0} \in \mathbb{R}$ and $T_{1}>0$ such that for all $n \in \mathbb{N}, \phi\left(-n T_{1}+T_{0}, W_{2}\right) \cap$ $W_{1} \neq \varnothing$. By (i) the periodic points are dense in $\mathscr{D}$, hence there are $\left(u_{j}, x_{j}\right) \in W_{j}$ with $\left(u_{j}, \varphi\left(\cdot, x_{j}, u_{j}\right)\right) t_{j}$-periodic for some $t_{j}>0, j=1,2$. It suffices again to take $W_{j}$ of the form $W_{j}=\left(V_{j} \times N_{j}\right) \cap \mathscr{D}$, with $\varphi\left(t, x_{j}, u_{j}\right) \in$ int $D$ for all $t \in \mathbb{R}, N_{j}=\left\{y \in M, d\left(x_{j}, y\right)<\varepsilon\right\}$, and

$$
V_{j}=\left\{v \in \mathscr{U},\left|\int_{\mathbb{R}}\left\langle u_{j}(\tau)-v(\tau), y_{i j}(\tau)\right\rangle d \tau\right|<\varepsilon\right\} \quad \text { for } \quad j=1,2
$$


There is $T>0$ such that for $j=1,2, i=1, \ldots, k_{j}$

$$
\int_{\mathbb{R} \backslash[-T, T]}\left|y_{i}(\tau)\right| d \tau<\frac{\varepsilon}{\operatorname{diam} U}
$$

We may assume that $t_{1}, t_{2}>T$. Furthermore, $\varphi\left(t, x_{j}, u_{j}\right) \in$ int $D$ for all $t \in \mathbb{R}$ implies that there exists $t_{0}>0$ and $v_{0} \in \mathscr{U}$ with $\varphi\left(t_{0}, x_{2}, v_{0}\right)=x_{1}$. Now fix $n \in \mathbb{N}$. Define $v_{n} \in \mathscr{U}$ through

$$
v_{n}(\tau)= \begin{cases}u_{1}(\tau) & \tau \in\left[-n t_{1}, \infty\right) \\ v_{0}\left(\tau+n t_{1}+t_{0}\right) & \tau \in\left[-n t_{1}-t_{0},-n t_{1}\right) \\ u_{2}\left(\tau+n t_{1}+t_{0}\right) & \tau \in\left(-\infty,-n t_{1}-t_{0}\right)\end{cases}
$$

Then $v_{n} \in V_{1}$, because

$$
\left|\int_{\mathbb{R}}\left\langle u_{1}(\tau)-v_{n}(\tau), y_{i 1}(\tau)\right\rangle d \tau\right| \leqslant \operatorname{diam} U \cdot \int_{\mathbb{R} \backslash\left[-t_{1}, t_{1}\right]}\left|y_{i 1}(\tau)\right| d \tau<\varepsilon
$$

Furthermore, $\varphi\left(t, x_{1}, v_{n}\right)=\varphi\left(t, x_{1}, u_{1}\right)$ for $t \geqslant-n t_{1}$ and $\varphi\left(t, x_{1}, v_{n}\right)=$ $\varphi\left(t+n t_{1}+t_{0}, x_{2}, u_{2}\right)$ for $t<-n t_{1}-t_{0}$. Therefore $\varphi\left(t, x_{1}, v_{n}\right) \in$ int $D$ for all $t \in \mathbb{R}$ and thus $\left(v_{n}, x_{1}\right) \in W_{1}$. On the other hand, observe that $\varphi\left(-n t_{1}-t_{0}-t_{2}, x_{1}, v_{n}\right)=x_{2}$ and $v_{n}\left(-n t_{1}-t_{0}-t_{2}+\tau\right)=u_{2}\left(\tau-t_{2}\right)=u_{2}(\tau)$ for $\tau<t_{2}$. Hence for all $i=1, \ldots, k_{2}$

$$
\begin{aligned}
\left|\int_{\mathbb{R}}\left\langle u_{2}(\tau)-v_{n}\left(-n t_{1}-t_{0}-t_{2}+\tau\right), y_{i 2}(\tau)\right\rangle d \tau\right| \\
\quad=\left|\int_{t_{2}}^{\infty}\left\langle u_{2}(\tau)-v_{n}\left(-n t_{1}-t_{0}-t_{2}+\tau\right), y_{i 2}(\tau)\right\rangle d \tau\right| \\
\quad \leqslant \operatorname{diam} U \cdot \int_{t_{2}}^{\infty}\left|y_{i 2}(\tau)\right| d \tau \\
\quad<\varepsilon
\end{aligned}
$$

Thus

$$
\begin{aligned}
& \phi\left(-n t_{1}-\left(t_{0}+t_{2}\right), v_{n}, x_{1}\right) \\
& \quad=\left(v_{n}\left(-n t_{1}-\left(t_{0}+t_{2}\right)+\cdot\right), \varphi\left(-n t_{1}-\left(t_{0}+t_{2}\right), x_{1}, v_{n}\right)\right) \\
& \quad=\left(\theta\left(-n t_{1}-\left(t_{0}+t_{2}\right), v_{n}\right), x_{2}\right) \in W_{2}
\end{aligned}
$$

(iii) In order to prove sensitive dependence on initial conditions, we need a metric on $\mathscr{U} \times M$. Any $p$-metric $d_{p}(p \in(0, \infty])$ on the product space 
with respect to $d_{\mathscr{U}}, d_{M}$, where $d_{\mathscr{U}}$ is the metric defined in (2.1) and $d_{M}$ is any Riemannian metric on $M$, yields immediately: If $d_{\mathscr{U}}(\theta(t, u), \theta(t, v)) \geqslant \delta$, then $d_{p}(\phi(t, u, x), \phi(t, v, x)) \geqslant \delta$. tion 3.5.

The following two lemmas present partial converses to Proposi-

3.6. Lemma. Let $(u, x) \in \mathscr{U} \times M$ be a $\phi$-periodic point. Then there exists a control set $D \subset M$ such that $x \in D$.

The proof follows immediately from the definitions. We will discuss similar relations with respect to recurrence and invariant measures in Sections 4 and 5 .

3.7. Lemma. Let $W \subset \mathscr{U} \times M$ be a closed, invariant set, such that $\phi_{\mid W}$ is topologically mixing. Then there exists a control set $D \subset M$ with

$$
\pi_{M} W=\{x \in M, \text { there exists } u \in \mathscr{U} \text { with }(u, x) \in W\} \subset D
$$

Proof. We have to show that for all $x, y \in \pi_{M} W$, there exists $u \in \mathscr{U}$ with $\varphi\left(t_{k}, x, u\right) \rightarrow y$ for a sequence $t_{k} \rightarrow \infty$. By assumption on $W$ there are, for every $\varepsilon>0$, arbitrarily large $t_{k}>0$ with $d\left(\varphi\left(t_{k}, x, u\right), y\right)<\varepsilon$.

The following main result of this section shows that there is a one-toone correspondence between control sets of (3.1) in $M$ and maximal topologically mixing sets in $\mathscr{U} \times M$.

3.8. Definition. Let $(S, \psi)$ be a dynamical system and $W \subset S$ a closed, $\psi$-invariant set such that $\psi_{\mid W}$ is topologically mixing. Then $W$ is called a maximal topologically mixing set, if every closed, $\psi$-invariant set $W^{\prime} \supset W$ with $\psi_{\mid W^{\prime}}$ topologically mixing, satisfies $W^{\prime}=W$.

3:9. Theorem. Assume that (H) holds. Let $\mathscr{D} \subset \mathscr{U} \times M$ with int $\pi_{M} \mathscr{D} \neq \varnothing$, where $\pi_{M} \mathscr{D}:=\{x \in M$, there exists $u \in \mathscr{U}$ with $(u, x) \in \mathscr{D}\}$.

Then $\mathscr{D}$ is maximal topologically mixing iff there exists a control set $D$ such that $\mathscr{D}$ is of the form (3.3).

In this case $D$ is unique and

$$
\text { int } D=\text { int } \pi_{M} \mathscr{D} \quad \text { and } \quad \operatorname{cl} D=\pi_{M} \mathscr{D}
$$

Proof. The set $\mathscr{D}$, defined by (3.3), is topologically mixing by Proposition 3.5. To prove the first assertion of (3.4), note that $\bar{D}=\overline{\operatorname{int}} \bar{D}$, and by the proof of Proposition 3.5(i), we have: For each $x \in$ int $D$ there exists a $u \in \mathscr{U}$ such that $\varphi(\cdot, x, u)$ is periodic and contained in int $D$. The second statement of (3.4) follows from (3.3). 
It remains to prove maximality of $\mathscr{D}$ : If $\mathscr{D}^{\prime} \supset \mathscr{D}$ is topologically mixing, then there exists $(u, x) \in \mathscr{D}^{\prime}$ with $\omega(u, x)=\mathscr{D}^{\prime}$ (see, e.g., Ref. 19, Proposition I.11.4). Thus there is a $t_{0} \in \mathbb{R}$ such that $\varphi(t, x, u) \in$ int $D$ for all $t \geqslant t_{0}$. Since $y:=\varphi\left(t_{0}, x, u\right) \in$ int $D$, there exists $v \in \mathscr{U}$ with $\varphi(t, y, v) \in \operatorname{int} D$ for all $t<0$. Define

$$
u^{0}(t)= \begin{cases}u(t) & t \geqslant t_{0} \\ v\left(t+t_{0}\right) & t<t_{0}\end{cases}
$$

then $\left(u^{0}, y\right) \in \mathscr{D}, \varphi\left(t, y, u^{0}\right) \in$ int $D$ for all $t \in \mathbb{R}$, and $\omega\left(u^{0}, y\right)=\omega(u, x)=\mathscr{D}^{\prime}$. Thus $\mathscr{D}^{\prime}=\omega\left(u^{0}, y\right) \subset \mathscr{D}$, because $\mathscr{D}$ is closed, and hence $\mathscr{D}$ is maximal.

To prove the "only if" statement, let $\mathscr{D}$ be a maximal topologically mixing set with int $\pi_{M} \mathscr{D} \neq \varnothing$. We first show that for $x_{1}, x_{2} \in$ int $\pi_{M} \mathscr{D}$ there are $u \in \mathscr{U}$ and $t>0$ with $\varphi\left(t, x_{1}, u\right)=x_{2}$. Let $y_{1} \in \operatorname{int} \mathscr{O}^{+}\left(x_{1}\right) \cap$ int $\pi_{M} \mathscr{D}$ and $y_{2} \in \operatorname{int} \mathcal{O}^{-}\left(x_{2}\right) \cap \operatorname{int} \pi_{M} \mathscr{D}$.

Consider neighborhoods $V_{1}$ of $y_{1}$ and $V_{2}$ of $y_{2}$ with $V_{1} \subset \operatorname{int} \mathscr{\theta}^{+}\left(x_{1}\right)$ and $V_{2} \subset$ int $\mathscr{O}^{-}\left(x_{2}\right)$. Since $\mathscr{D}$ is also topologically transitive, there exists $(u, x)$ with $\omega(u, x)=\mathscr{D}$. Thus a point in $V_{2}$ can be reached from a point in $V_{1}$, and hence $x_{2}$ can be reached from $x_{1}$ as claimed. This implies that there is a control set $D$ with int $\pi_{M} \mathscr{D} \subset$ int $D$. As in (3.3), $D$ can be lifted to a maximal topologically mixing set $\tilde{\mathscr{D}}$. We claim that $\mathscr{\mathscr { D }} \subset \mathscr{D}$; then maximality of $\tilde{\mathscr{D}}$ proves $\mathscr{D}=\widetilde{\mathscr{D}}$ as desired. Since $\omega(u, x)=\mathscr{D}$ and int $\pi_{M} \mathscr{D} \subset$ int $D=$ int $\pi_{M} \widetilde{\mathscr{D}}$, one finds as in (i), $\left(u^{0}, y\right) \in \widetilde{\mathscr{D}}$ with $\omega\left(u^{0}, y\right)=\mathscr{D}$. Now invariance of $\tilde{\mathscr{D}}$ implies $\mathscr{\mathscr { D }} \subset \mathscr{D}$.

Actually the proof given above only uses topological transitivity. Therefore one obtains another dynamical systems interpretation of control sets.

3.10. Corollary. Control sets $D \subset M$ of (3.1) with int $D \neq \varnothing$ uniquely correspond to maximal topologically transitive sets $\mathscr{D}$ with int $\pi_{M} \mathscr{D} \neq \varnothing$ via (3.3).

Theorem 3.9 and its corollary show, in particular, that the maximal topologically transitive and the maximal topologically mixing sets of the dynamical system (3.2) coincide and that they are already characterized by the control property of the control system (3.1) on $M$. One therefore obtains the following.

3.11. Corollary. Assume that $(H)$ holds. Then the following statements are equivalent: 
(i) The control system (3.1) is completely controllable on $M$.

(ii) The dynamical system $(\mathscr{U} \times M, \phi)$, defined by (3.2), is topologically mixing.

(iii) The dynamical system $(\mathscr{U} \times M, \phi)$ is topologically transitive.

\section{RECURRENCE AND CHAIN RECURRENCE, CONTROL SETS, AND CHAIN CONTROL SETS}

In this section we analyze recurrence and chain recurrence of the shift space $(\mathscr{U}, \theta)$ from Section 2 and of the dynamical system (3.2). It will turn out that the $M$-component of recurrent points of $(\mathscr{U} \times M, \phi)$ can be characterized via control sets $D \subset M$, but conversely, for a control set $D$ the lift to $\mathscr{D} \subset \mathscr{U} \times M$ is, in general, not recurrent. For the more general concept of chain recurrence and associated chain control sets, however, we obtain that the components of the chain recurrent set are the lifts of chain control sets.

4.1. Definition. Let $(S, \psi)$ be a dynamical system. A point $x \in S$ is said to be recurrent if $x \in \omega(x)$, the $\omega$-limit set of $x$ under $\psi .(S, \psi)$ is called recurrent, if all $x \in S$ are recurrent.

For the dynamical system (3.2) we have seen in Section 3: If $D \subset M$ is a control set of the control system (3.1) with int $D \neq \varnothing$, then there exists $(u, x) \in \mathscr{D}$ such that $\omega(u, x)=\mathscr{D}$ [proof of Theorem 3.9(ii)]. Since $\phi_{1 \mathscr{D}}$ is topologically transitive, this implies that there exists a residual subset of points $(u, x) \in \mathscr{D}$ such that $\omega(u, x)=\mathscr{D}$ (see, e.g., Ref. 19, Proposition I.11.4). Furthermore, the periodic, hence recurrent, points are dense in $\mathscr{D}$ [Proposition 3.5(i)]. But since the shift $\theta$ is not recurrent on $\mathscr{U}$, if $U$ consists of more than one point, the dynamical system $\left(\mathscr{D}, \phi_{\mid \mathscr{D}}\right)$ is not recurrent: Take, e.g., the situation where the control system (3.1) is completely controllable, i.e., $M=D$, but $(\mathscr{U} \times M, \phi)$ is not recurrent, if $U$ contains more than one point.

We have however, the following characterization of the $M$-component of $\phi$-recurrent points:

4.2. Lemma. A point $x \in M$ is an element of some control set $D$ of (3.1) iff there exists $u \in \mathscr{U}$ such that $x \in \pi_{M} \omega(u, x)$, i.e., in particular, if $(u, x)$ is recurrent.

Proof. Assume first that $x \in \pi_{M} \omega(u, x)$ for some $u \in \mathscr{U}$, and pick $y:=\varphi(t, x, u)$ for some $t>0$. Then $y \in \mathcal{O}^{+}(x)$ and $x \in \overline{\mathcal{O}^{+}(y)} \subset \overline{\mathcal{O}^{+}(x)}$, i.e., $x$ and $y$ are contained in the same control set.

Vice versa, let $x \in D$, some control set, and choose a sequence $\varepsilon_{n} \rightarrow 0$. We construct a control $u: \mathbb{R} \rightarrow U$ in the following way: Denote by $B(x, \varepsilon)$ 
the open ball of radius $\varepsilon$ in $M$, centered at $x$. Since $x \in D$, there exist $x_{1} \in D \cap B\left(x, \varepsilon_{1}\right), t_{1}>0$, and $u_{1} \in \mathscr{U}$ such that $\varphi\left(t_{1}, x, u_{1}\right)=x_{1}$. Now $x, x_{1} \in D$, and hence there are $x_{2} \in D \cap B\left(x, \varepsilon_{2}\right), t_{2}>0$, and $u_{2} \in \mathscr{U}$ such that $\varphi\left(t_{2}, x_{1}, u_{2}\right)=x_{2}$, etc., for all $n \in \mathbb{N}$. Define (with $t_{0}=0$ )

$$
u(t)=u_{n}\left(t-\sum_{i=0}^{n-1} t_{i}\right) \quad \text { for } \quad t \in\left(\sum_{i=0}^{n-1} t_{i}, \sum_{i=0}^{n} t_{i}\right]
$$

and arbitrary for $t \leqslant 0$. We may choose $\left(t_{i}\right)$ such that $\sum_{i=0}^{\infty} t_{i}=\infty$. Then $x \in \pi_{M} \omega(u, x)$.

Note that, if the control system (3.1) is completely controllable on $M$, then for all $x \in M$ there exists $u \in \mathscr{U}$ such that $(u, x) \in \mathscr{U} \times M$ is recurrent (even periodic). But the converse is not true in general, even under assumption $(\mathrm{H})$ and for compact $M$, as the following example shows.

4.3. Example. Consider the following control system on the projective space $\mathbb{P}^{1}$ :

$$
\dot{x}(t)=u_{0}(t) \cos (2 x(t))+u_{1}(t) f_{1}(x(t))+u_{2}(t) f_{2}(x(t)), \quad x \in \mathbb{R} \bmod \pi
$$

with $U=[0,1]^{3}$, where $f_{1}(x), f_{2}(x)$ are $C^{\infty}$ such that

$$
\begin{aligned}
& f_{1}(x) \begin{cases}>0 & x \in\left(\frac{\pi}{8}, \frac{\pi}{2}\right) \\
=0 & x=\frac{\pi}{2} \\
>0 & x \in\left(\frac{\pi}{2}, \frac{7 \pi}{8}\right) \\
=0 & \text { otherwise in }[0, \pi)\end{cases} \\
& f_{2}(x) \begin{cases}=0 & x=0 \\
<0 & x \in\left(0, \frac{3 \pi}{8}\right) \\
<0 & x \in\left(\frac{5 \pi}{8}, \pi\right) \\
=0 & \text { otherwise in }[0, \pi)\end{cases}
\end{aligned}
$$

Then there are two control sets $D_{1}=[0, \pi / 2], D_{2}=(\pi / 2, \pi)$. All points $x \in \mathbb{P} \backslash\{0, \pi / 2\}$ are in the interior of some control set, and hence for these 
$x$ there exists $u \in \mathscr{U}$, such that $(u, x)$ is periodic, by the proof of Proposition 3.5(i). For $x=0, \pi / 2$ choose $u_{0}(t) \equiv 0$, and $\left(u_{1}(t), u_{2}(t)\right) \in[0,1]^{2}$ arbitrary but periodic. Then $\varphi(t, x, u)=x$ for $x=0$ or $x=\pi / 2$. Hence for every $x \in \mathbb{P}^{1}$ there is $u \in \mathscr{U}$ such that $(u, x)$ is $\phi$-recurrent.

Recall that recurrence is related to invariant measures and their support, e.g., via Poincaré's recurrence theorem. We investigate their connection in Section 5.

We have seen that, although the $M$-component of recurrent points can be characterized via control sets on $M$, these sets cannot be lifted to recurrent components in $\mathscr{U} \times M$ because the shift space $(\mathscr{U}, \theta)$ is not recurrent. For the more general concept of chain recurrence we do obtain a correspondence with chain control sets on $M$.

4.4. Definition. Let $(S, \psi)$ be a dynamical system on a metric space $(S, d)$. For $\varepsilon>0$ and $T>0$ an $(\varepsilon, T)$-chain from $x \in S$ to $y \in S$ consists of a sequence $x_{0}, \ldots, x_{k}$ in $S$ and a sequence $t_{0}, \ldots, t_{k-1}$ in $\mathbb{R}$ such that $x_{0}=x$, $x_{k}=y, t_{j} \geqslant T$ and

$$
d\left(\psi\left(t_{j}, x_{j}\right), x_{j+1}\right) \leqslant \varepsilon \quad \text { for } \quad j=0, \ldots, k-1
$$

For $X \subset S$ define the chain-limit set by

$$
\begin{array}{ll}
\Omega(X)=\{y \in S, & \text { for all } \varepsilon>0 \text { and all } T>0 \text { there exists } x \in X \text { such that } \\
\text { there is an }(\varepsilon, T) \text {-chain from } x \text { to } y\}
\end{array}
$$

and the chain-recurrent set as

$$
\mathscr{C} \mathscr{R}=\{x \in S, x \in \Omega(x)\}
$$

The dynamical system $(S, \psi)$ is called chain recurrent if $S=\mathscr{C} \mathscr{R}$, and chain transitive, if $y \in \Omega(x)$ for all $x, y \in S$. Recall that $(S, \psi)$ is chain transitive iff it is chain recurrent and $S$ is connected, and for $X$ closed, $\Omega(X)$ is closed and invariant and contains $\omega(x)$ for all $x \in X$.

\subsection{Lemma. The shift $(\mathscr{U}, \theta)$ is chain transitive.}

Proof. By Lemma 2.2 the periodic points are dense in $\mathscr{U}$. Since periodic points are chain recurrent, and the chain recurrent set is closed (see, e.g., Ref. 10, II.6.A), it follows that $(\mathscr{U}, \theta)$ is chain recurrent. Because $\mathscr{U}$ is also connected, it is chain transitive.

4.6. Definition. For the control system (3.1) a set $E \subset M$ is called a chain control set, if 
(i) for all $x, y \in E$ and all $\varepsilon, T>0$, there are $k \in \mathbb{N}, x=x_{0}, \ldots, x_{k}=y$ in $M, t_{0}, \ldots, t_{k-1} \geqslant T$, and $u_{0}, \ldots, u_{k-1}$ in $\mathscr{U}$ with

$$
d\left(\varphi\left(t_{j}, x_{j}, u_{j}\right), x_{j+1}\right)<\varepsilon \quad \text { for } \quad j=0, \ldots, k-1
$$

(ii) for all $x \in E$ there exists $u \in \mathscr{U}$ such that $\varphi(t, x, u) \in E$ for all $t \in \mathbb{R}$, and

(iii) $E$ is maximal with properties (i) and (ii).

4.7. Lemma. Chain control sets are closed, connected, and pairwise disjoint.

Proof. Closedness and connectedness follow directly from the definition. To show disjointness, assume that $x \in E_{1} \cap E_{2}$, and pick $y_{i} \in E_{i}$, $i=1,2$. Then for all $\varepsilon>0, T>0$, there exists $k_{i} \in \mathbb{N}, x_{0}, \ldots, x_{k_{1}}, z_{0}, \ldots, z_{k_{2}}$ in $M, u_{0}, \ldots, u_{k_{1}-1}, v_{0}, \ldots, v_{k_{2}-1}$, and $t_{0}, \ldots, t_{k_{1}-1}, s_{0}, \ldots, s_{k_{2}-1} \geqslant T$ with $x_{0}=y_{1}$, $x_{k}=x=z_{0}, z_{k_{2}}=y_{2}$, and

$$
\begin{array}{ll}
d\left(\varphi\left(t_{j}, x_{j}, u_{j}\right), x_{j+1}\right)<\varepsilon & \text { for } j=0, \ldots, k_{1}-1, \\
d\left(\varphi\left(s_{j}, z_{j}, v_{j}\right), z_{j+1}\right)<\varepsilon & \text { for } j=0, \ldots, k_{2}-1,
\end{array}
$$

By maximality of chain control sets, we have $E_{1}=E_{2}$.

For a chain control set $E \subset M$, define [compare (3.3)]

$$
\mathscr{E}=\{(u, x) \in \mathscr{U} \times M, \varphi(t, x, u) \in E \text { for all } t \in \mathbb{R}\}
$$

\subsection{Theorem.}

(i) Let $E \subset M$ be a chain control set. Then $\mathscr{E} \subset \mathscr{U} \times M$ as defined by (4.2) is a maximal invariant chain transitive set for the system $(\mathscr{U} \times M, \phi)$.

(ii) Let $\mathscr{E} \subset \mathscr{U} \times M$ be a maximal invariant chain transitive set in $(\mathscr{U} \times M, \phi)$. Then $\pi_{M} \mathscr{E}$ is a chain control set.

Proof. (i) Let $(u, x),(v, y) \in \mathscr{E}$ and pick $\varepsilon>0, T>0$. Recall the definition of the metric $d_{\mathscr{U}}$ on $\mathscr{U}$ given in (2.1) and choose $N \in \mathbb{N}$ large enough such that $\sum_{n=N+1}^{\infty} 2^{-n}<\varepsilon / 2$. For finitely many $y_{1}, \ldots, y_{N} \in$ $L^{1}\left(\mathbb{R}, \mathbb{R}^{m}\right)$, there exists $\hat{T}>0$ such that

$$
\int_{\mathbb{R} \backslash[-\hat{T}, \hat{T}]}\left|y_{i}(\tau)\right| d \tau<\frac{\varepsilon}{2 \operatorname{diam} U} \quad \text { for } \quad i=1, \ldots, N
$$

Since the choice of $\hat{T}$ is independent of the $x_{j}, u_{j}$ above, we can assume w.l.o.g. that $T>\hat{T}$. Chain controllability from $\varphi(2 T, x, u) \in E$ to $\varphi(-T, y, v) \in E$ yields the existence of $k \in \mathbb{N}$ and $x_{0}, x_{1}, \ldots, x_{k} \in M$, $u_{0}, \ldots, u_{k-1} \in \mathscr{U}, t_{0}, \ldots, t_{k-1} \geqslant T$ with $x_{0}=\varphi(2 T, x, u), x_{k}=\varphi(-T, y, v)$ and $d\left(\varphi\left(t_{j}, x_{j}, u_{j}\right), x_{j+1}\right)<\varepsilon$ for $j=0, \ldots, k-1$. 
We now construct an $(\varepsilon, T)$-chain from $(u, x)$ to $(v, y)$ in the following way:

Let

$$
\begin{aligned}
t_{-2} & =T, & x_{-2} & =x, \\
t_{-1} & =T, & v_{-2} & =u \\
& x_{-1}=\varphi(T, x, u), & v_{-1}(t) & = \begin{cases}u\left(t_{-2}+t\right), & t \leqslant t_{-1} \\
u_{0}\left(t-t_{-1}\right), & t>t_{-1}\end{cases}
\end{aligned}
$$

the times $t_{0}, \ldots, t_{k-1}$ and the points $x_{0}, \ldots, x_{k}$ are as above. Furthermore, set

$$
t_{k}=T, \quad x_{k+1}=y, \quad v_{k+1}=v
$$

and define for $j=0, \ldots, k-2$,

$$
v_{j}(t)= \begin{cases}v_{j-1}\left(t_{j-1}+t\right), & t \leqslant 0 \\ u_{j}(t), & 0<t<t_{j} \\ u_{j+1}\left(t-t_{j}\right), & t>t_{j}\end{cases}
$$

and

$$
\begin{aligned}
v_{k-1}(t) & = \begin{cases}v_{k-2}\left(t_{k-2}+t\right), & t \leqslant 0 \\
u_{k-1}(t), & 0<t<t_{k-1} \\
v_{k}\left(t-t_{k-1}-T\right), & t>t_{k-1}\end{cases} \\
v_{k}(t) & = \begin{cases}v_{k-1}\left(t_{k-1}+t\right), & t \leqslant 0 \\
v(t-T), & t>0\end{cases}
\end{aligned}
$$

It is easily seen that

$$
\left(v_{-2}, x_{-2}\right),\left(v_{-1}, x_{-1}\right), \ldots,\left(v_{k+1}, x_{k+1}\right), \text { and } t_{-2}, t_{-1}, \ldots, t_{k} \geqslant T
$$

is an $(\varepsilon, T)$-chain from $(u, x)$ to $(v, y)$ provided that for $j=-2,-1, \ldots, k$,

$$
d\left(v_{j}\left(t_{j}+\cdot\right), v_{j+1}\right)<\varepsilon
$$

By choice of $\hat{T}$ and $N$, one has for all $w_{1}, w_{2} \in \mathscr{U}$,

$$
\begin{aligned}
d\left(w_{1}, w_{2}\right)= & \sum_{n=1}^{\infty} 2^{-n} \frac{\left|\left\langle w_{1}-w_{2}, y_{n}\right\rangle\right|}{1+\left|\left\langle w_{1}-w_{2}, y_{n}\right\rangle\right|} \\
\leqslant & \sum_{n=1}^{N} 2^{-n}\left\{\left|\int_{\mathbb{R} \backslash[-\hat{T}, \hat{T}]}\left\langle w_{1}(t)-w_{2}(t), y_{n}(t)\right\rangle d t\right|\right. \\
& \left.+\left|\int_{-\hat{T}}^{\hat{T}}\left\langle w_{1}(t)-w_{2}(t), y_{n}(t)\right\rangle d t\right|\right\}+\frac{\varepsilon}{2} \\
< & +\max _{n=1, \ldots, N} \int_{-\hat{T}}^{\hat{T}}\left|w_{1}(t)-w_{2}(t)\right|\left|y_{n}(t)\right| d t
\end{aligned}
$$


Hence it suffices to show that for all considered pairs of control functions the integrand in the integral above vanishes. This is immediate from the definition of $v_{j}, j=-2, \ldots, k+1$.

(ii) Let $\mathscr{E}$ be an invariant, chain transitive set in $\mathscr{U} \times M$. For $x \in \pi_{M} \mathscr{E}$ there exists $u \in \mathscr{U}$ such that $\varphi(t, x, u) \in \mathscr{E}$ for all $t \in \mathbb{R}$ by invariance. Now let $x, y \in \pi_{M} \mathscr{E}$ and choose $\varepsilon>0, T>0$. Then by chain transitivity of $\mathscr{E}$, we can choose $x_{j}, u_{j}, t_{j}$ such that (4.1) holds.

The proof of (i) and (ii) is concluded by the observation that $E$ is maximal iff $\mathscr{E}$ is maximal.

4.9. Remark. Theorem 4.8 identifies the lifted chain control sets $\mathscr{E} \subset \mathscr{U} \times M$ as the components of the chain recurrent set $\mathscr{C} \mathscr{R} \subset \mathscr{U} \times M$. On the other hand, $\mathscr{C} \mathscr{R}=\bigcap\left\{A \cup A^{*}, A\right.$ is an attractor of $(\mathscr{U} \times M, \phi)$ and $A^{*}$ its complementary repeller $\}$; see Conley (10), II.6.2. This leads to a characterization of the $\mathscr{E}$ 's as the Morse sets of $(\mathscr{U} \times M, \phi)$, where Morse sets are intersections of attractors and repellers; see Conley (10), II.7. This relation between chain control sets in $M$ and (finest) Morse decompositions in $\mathscr{U} \times M$ is investigated in Ref. 7 for flows on vector bundles.

Theorems 3.9 and 4.8 suggest to find conditions under which control sets (or their closures) coincide with chain control sets. Note that for each control set $D \subset M$, there exists a unique chain control set $E \subset M$ such that $\bar{D} \subset E$. But if $D_{1}, D_{2}$ are control sets with $\overline{D_{1}} \cap \overline{D_{2}} \neq \varnothing$, then there is one chain control set $E$ with $\overline{D_{1}} \cup \overline{D_{2}} \subset E$, i.e., in general the closures of control sets need not be chain control sets.

For general nonlinear control systems and their control sets, little can be said about this problem in terms of orbits and control properties. In terms of chain orbits we have the following, almost trivial observation: Let $D$ be a control set; then $\bar{D}$ is a chain control set iff $\Omega(\mathscr{D}) \cap \Omega^{*}(\mathscr{D})=\mathscr{D}$, where $\Omega^{*}(X)$ is defined as $\Omega(X)$ in Definition 4.4, but backward in time [compare also Conley (10), II.6]. For specific classes of control systems one can be more precise; compare, e.g., Ref. 7 for control systems on projective spaces, associated with linear semigroups.

Note that under assumption $(\mathrm{H})$ a control set $D$ is closed iff $D$ is invariant, i.e., $\overline{\mathcal{O}^{+}(x)}=\bar{D}$ for all $x \in D$. Then invariant control sets have nonvoid interior, and on a compact manifold $M$ the control system (3.1) has at least one invariant control set $C$. Closed control sets $C$ are chain control sets, if each $x \in \partial C$ has a neighborhood $N(x)$ such that for all $y \in N(x)$ and all $u \in \mathscr{U}$, there exists $t \geqslant 0$ such that $\varphi(t, y, u) \in C$, i.e., if $C$ is isolated (compare the definition of isolated sets for flows in Ref. 10.

The following examples show that several control sets, even with nonvoid interior, can be contained in one chain control set and that chain 
control sets may contain points which are in no control set, if the control sets are not isolated.

4.10. Example. On the circle $S^{1}$ consider the control system

$$
\dot{x}(t)=-\sin ^{2} x(t)+a \cos ^{2} x(t)-u(t) \cos ^{2} x(t), \quad x \in \mathbb{R} \bmod 2 \pi
$$

with $a>0$ and $U=[A, a] \subset \mathbb{R}$. Arnold and Kliemann (1) have described a general procedure to compute control sets for systems with one-dimensional state space. As a consequence we obtain for this example: There are four control sets $D_{1}=\left[0, \arctan (a-A)^{1 / 2}\right], D_{2}=\left(\pi-\arctan (a-A)^{1 / 2}, \pi\right)$, $D_{3}=D_{1}+\pi, D_{4}=D_{2}+\pi$. There is, however, exactly one chain control set $E=\mathbb{S}^{1}$. Note that $D_{1} \cap \overline{D_{4}} \neq \varnothing, \overline{D_{2}} \cap D_{3} \neq \varnothing$, and points in $\mathbb{S}^{1} \backslash\left(\bigcup_{i=1}^{4} \overline{D_{i}}\right)$ $\neq \varnothing$ are contained in no control set, but in $E$.

4.11. Example. Again on $\mathbb{S}^{1}$ consider the control system

$$
\begin{gathered}
\dot{x}(t)=\frac{1}{2} \sin 2 x(t)+u_{1}(t)\left[\cos ^{2} x(t)-\sin ^{2} x(t)\right] \\
-u_{2}(t) \sin 2 x(t), \quad x \in \mathbb{R} \bmod 2 \pi
\end{gathered}
$$

with $U=\left[0, \frac{1}{2}\right] \times\left[\frac{1}{2}, 1\right]$. The control sets are in this case $D_{1}=[\pi / 4, \pi / 2]$, $D_{2}=(3 \pi / 4, \pi), D_{3}=D_{1}+\pi, D_{4}=D_{2}+\pi$, and $D_{\alpha}=\{\alpha\}, \alpha \in \mathbb{S}^{1} \backslash\left(\bigcup_{i=1}^{4} D_{i}\right)$. Note that int $D_{i} \neq \varnothing$ for $i=1, \ldots, 4$ and $\overline{D_{i}} \cap \overline{D_{j}}=\varnothing$ for $i \neq j, i, j \in\{1, \ldots, 4\}$. But the only chain control set is $E=\mathbb{S}^{1}$.

\section{INVARIANT MEASURES OF $(\mathscr{U} \times M, \phi)$ AND THEIR SUPPORT}

Assume from now on that $M$ is compact. This condition is not really needed for all of the results that follow, but for existence results of invariant measures one needs some form of tightness (in order to have weak limits of certain families of probability measures), and the most convenient way to assure this is the hypothesis of compactness for $M$. First we characterize the $\phi$-invariant measures.

5.1. Definition. Let $(S, \psi)$ be a dynamical system. A probability measure $\mu$ on $S$ is called $\psi$-invariant, if $\psi_{t} \mu=\mu$ for all $t \in \mathbb{R}$. We denote

$$
\mathscr{M}_{\mu}=\{\mu \text { probability measure on } S, \mu \text { is } \psi \text {-invariant }\} \text {. }
$$


5.2. Proposition. A probability measure $\mu$ on $\mathscr{U} \times M$ is $\phi$-invariant iff $\mu$ is of the form

$$
\mu(d u, d x)=\mu_{u}(d x) \rho(d u)
$$

where $\rho \in \mathscr{M}_{\theta}$ and $\varphi(t, \cdot, u) \mu_{u}=\mu_{\theta_{t} u} \rho$-a.s. for $t \in \mathbb{R}$.

Proof. This follows from Crauel (11), Lemma 2; we include the proof for the sake of completeness. Note first that the $\mathscr{U}$-component of $\phi$ (i.e., the shift $\theta$ ) is decoupled, and thus we can consider it separately. If $\mu$ is $\phi$-invariant, then its marginal $\pi_{\mathscr{U}} \mu$ on $\mathscr{U}$ is $\theta$-invariant. Every $\mu$ desintegrates as $\mu(d u, d x)=\mu_{u}(d x) \rho(d u)$, where $\rho=\pi_{\mathscr{U}} \mu$ [cf. Gänssler and Stute (17); Satz 5.3.2.1]. If $\bar{\mu}_{u}$ denotes the desintegration of $\phi_{t} \mu$ with respect to $\rho=\pi_{\mathscr{U}} \mu$, and $\rho$ is $\theta$-invariant, then

$$
\bar{\mu}_{u}=\varphi(-t, \cdot, u)^{-1} \mu_{\theta_{-t} u}
$$

For information on the support of $\mu \in M_{\phi}$ we will use the KrylovBogolyubov construction. This requires some additional results on $\omega$-limit sets of $\phi$.

\subsection{Lemma.}

(i) For all $(u, x) \in \mathscr{U} \times M$ the limit sets $\omega(u, x)$ are connected, compact, and $\phi$-invariant, hence they contain minimal $\phi$-invariant sets.

(ii) For all $(u, x) \in \mathscr{U} \times M$ there exists a control set $D$ of (3.1) such that $\pi_{M} \omega(u, x) \cap D \neq \varnothing$.

(iii) For all $(u, x) \in \mathscr{U} \times M$ there exists a chain control set $E$ of (3.1) such that $\pi_{M} \omega(u, x) \subset E$.

(iv) Let $W \subset \mathscr{U} \times M$ be a minimal, $\phi$-invariant set; then there exists a control set $D$ of (3.1) such that $\pi_{M} W \subset D$.

(v) Let $D \subset M$ be a control set of (3.1); then for any $y \in \bar{D}$ there exists $(u, x) \in \mathscr{U} \times M$ such that $y \in \pi_{M} \omega(u, x)$.

Proof. (i) Is a standard property of dynamical systems.

(iii) Follows immediately from the definitions.

(iv) Each trajectory $\left\{\phi_{t}(u, x), t \geqslant 0\right\}$ in a minimal, $\phi$-invariant set $W$ is dense in $W$. Now take $x_{1}, x_{2} \in \pi_{M} W$, i.e., there exist $u_{1}, u_{2} \in \mathscr{U}$ such that $\left(u_{1}, x_{1}\right),\left(u_{2}, x_{2}\right) \in W$. Then for each $\varepsilon>0$ there exist $T_{1}, T_{2}>0$ such that $d_{M}\left(\varphi\left(T_{1}, x_{1}, u_{1}\right), x_{2}\right)<\varepsilon$ and $d_{M}\left(\varphi\left(T_{2}, x_{2}, u_{2}\right), x_{1}\right)<\varepsilon$, i.e., $x_{1}$ and $x_{2}$ are in one control set of (3.1).

(ii) Is an immediate consequence of (i) and (iv). 
(v) If $D$ consists of a single point, the result is obvious. In the other case, fix $y \in \bar{D}$ and consider a positive sequence $\varepsilon_{n} \rightarrow 0$. Pick $x \in D$; then there exist $u_{1} \in \mathscr{U}$ and $t_{1}>0$ such that $d_{M}\left(\varphi\left(t_{1}, x, u_{1}\right), y\right)<\varepsilon_{1}$, and $\varphi\left(t_{1}, x, u_{1}\right)=: x_{1} \in D$. For $x_{1}$ there are $u_{2} \in \mathscr{U}$ and $t_{2}>0$ with $d_{M}\left(\varphi\left(t_{2}, x_{1}, u_{2}\right), y\right)<\varepsilon_{2}$, and $\varphi\left(t_{2}, x_{1}, u_{2}\right)=: x_{2} \in D$, etc. Now define a control,

$$
u(t)=u_{k}\left(t-\sum_{i=1}^{k-1} t_{i}\right) \quad \text { for } \quad t \in\left[\sum_{i=1}^{k-1} t_{i}, \sum_{i=1}^{k} t_{i}\right)
$$

where we can assume, as in the proof of Lemma 4.2, that $\sum t_{i} \rightarrow \infty$. Then the point $(u, x) \in \mathscr{U} \times M$ satisfies $y \in \pi_{M} \omega(u, x)$ by construction.

\subsection{Remark.}

(i) If the control set $D \subset M$ satisfies int $D \neq \varnothing$, then for any $x \in$ int $D$, there is a minimal invariant set $W \subset \mathscr{U} \times M$ with $x \in \pi_{M} W$ : Just pick a periodic control function $u \in \mathscr{U}$ with periodic solution $\varphi(\cdot, x, u) \subset$ int $D$; see the proof of Proposition $3.5(i)$.

(ii) While all points in control sets of (3.1) correspond to some $\omega$-limit set by Lemma 5.3(v), this need not be true for points in chain control sets: Consider Example 4.10. There all points in $\mathbb{S}^{1} \backslash\left(\bigcup_{i=1}^{4} \bar{D}_{i}\right)$ are contained in the chain control set $E=\mathbb{S}^{1}$, but they cannot belong to any projection of an $\omega$-limit set $\omega(u, x) \subset$ $\mathscr{U} \times M$.

Now we can construct $\phi$-invariant probability measures on $\mathscr{U} \times M$ via the Krylov-Bogolyubov device, i.e., for $(u, x) \in \mathscr{U} \times M$ consider the Cesaro limits for sequences $t_{k} \rightarrow \infty$

$$
\lim _{\tau_{k} \rightarrow \infty} \frac{1}{t_{k}} \int_{0}^{t_{k}} F\left(\theta_{\tau}(u), \varphi(\tau, x, u)\right) d \tau=\int_{\mathscr{U} \times M} F(v, y) d \mu_{u, x}
$$

for all $F \in C(\mathscr{U} \times M, \mathbb{R})$, the continuous functions from $\mathscr{U} \times M$ into $\mathbb{R}$. The following properties of the probability measures $\mu_{u, x}$ are well-known; e.g., Ref, 19, Chapter II.6.

(a) $\mathscr{M}_{\theta}$ and $\mathscr{M}_{\phi}$ are nonempty.

(b) Define

$$
\begin{aligned}
\Sigma_{\phi}^{e} & =\left\{(u, x) \in \mathscr{U} \times M, \mu_{u, x} \text { as defined in (5.1) is ergodic }\right\} \\
\Sigma_{\phi}^{s} & =\left\{(u, x) \in \Sigma_{\phi}^{e},(u, x) \in \operatorname{supp} \mu_{u, x}\right\}
\end{aligned}
$$


then $\Sigma_{\phi}^{s} \neq \varnothing$, and $\Sigma_{\phi}^{s}$ has total measure with respect to $\mathscr{M}_{\phi}$, i.e., $\mu\left(\Sigma_{\phi}^{s}\right)^{c}=0$ for all $\mu \in \mathscr{M}_{\phi}$. Here supp $\mu$ stands for the support of the measure $\mu$, and $A^{c}$ denotes the complement of the set $A$.

(c) Each $\mu \in \mathscr{M}_{\phi}$ has an ergodic decomposition: Each $F \in$ $L^{1}(\mathscr{U} \times M, \mu)$ is $\mu_{u, x}$-integrable for $\mu$-almost all $(u, x) \in \Sigma_{\phi}^{s}$ and

$$
\int\left(\int_{\mathscr{U} \times M} F d \mu_{\mu, x}\right) d \mu=\int_{\mathscr{U} \times M} F d \mu
$$

According to (a)-(c), if we want to characterize the points that can belong to the support of some $\mu \in \mathscr{M}_{\phi}$, it suffices to characterize the set $\Sigma_{\phi}^{s}$.

\subsection{Theorem.}

(i) For all $(u, x) \in \mathscr{U} \times M$ we have $\operatorname{supp} \mu_{u, x} \subset \omega(u, x)$.

(ii) For all $(u, x) \in \mathscr{U} \times M$ there exists $\Gamma \subset \operatorname{supp} \mu_{u, x}$ with $\mu_{u, x} \Gamma=1$, such that for all $(v, y) \in \Gamma$ there is a control set $D \subset M$ with $\varphi(t, y, v) \subset D \cap \pi_{M} \omega(u, x)$, for all $t \geqslant 0$.

(iii) If $(u, x) \in \Sigma_{\phi}^{e}$, then there exists a (unique) control set $D \subset M$ such that for $\mu_{u, x}$-almost all $(v, y)$, we have $\varphi(\cdot, y, v) \subset D$, i.e., $\operatorname{supp} \mu_{u, x} \subset \mathscr{D}^{+}$, with

$$
\mathscr{D}^{+}=\operatorname{cl}\{(u, x) \in \mathscr{U} \times M, \varphi(t, x, u) \in D \text { for all } t \geqslant 0\}
$$

compare (3.3).

(iv) Let $D \subset M$ be a control set with int $D \neq \varnothing$. Then for each $x \in$ int $D$ there exists $u \in \mathscr{U}$ such that $(u, x) \in \Sigma_{\phi}^{s}$.

Proof. (i) Let $W \subset \mathscr{U} \times M$ be a neighborhood of $\omega(u, x)$; then there is a $T>0$ such that $\phi_{t}(u, x) \in W$ for all $t \geqslant T$, i.e., supp $\mu_{u, x} \subset W$.

(ii) By Poincaré's recurrence theorem, for every $(u, x) \in \mathscr{U} \times M$, there is a set $\Gamma \subset \mathscr{U} \times M$ with $\mu_{x, u} \Gamma=1$, such that for all $(v, y) \in \Gamma$ the trajectory $\{\phi(t, v, y), t \geqslant 0\}$ is recurrent; see, e.g., Ref. 19, Theorem I.2.3. By (i) we can assume that $\Gamma \subset \omega(u, x)$. But then the trajectory $\varphi(\cdot, y, v)$ on $M$ is contained in some control set $D$ : For all $t>0$ we have $\varphi(t, y, v) \in \mathcal{O}^{+}(y)$ and $y \in \overline{\mathcal{O}^{+}(\varphi(t, y, v))}$ by recurrence. Since $\omega(u, x)$ is $\phi$-invariant, we have $\varphi(t, y, v) \subset D \cap \pi_{M} \omega(u, x)$ for all $t \geqslant 0$.

(iii) Recall that $\mu$ is ergodic, if all $\phi$-invariant sets have $\mu$-measure 1 or 0 . Therefore for $\mu_{u, x}$-almost all $(v, y)$ we have $\omega(v, y)=\operatorname{supp} \mu_{u, x}$, and supp $\mu_{u, x}$ is a minimal set. Thus the control set, constructed in (ii), is the same for all $(v, y)$ with $\omega(v, y)=\operatorname{supp} \mu_{u, x}$. 
(iv) For $x \in$ int $D$ we construct $u \in \mathscr{U}$ as in the proof of Proposition 3.5(i) such that $\phi_{t}(u, x)$ has minimal period $T>0$, i.e., $\varphi(t, x, u) \neq x$ for all $t \in(0, T)$. The trajectory $\left\{\phi_{t}(u, x), t \in \mathbb{R}\right\}$ determines an invariant measure $\mu_{u, x}$ in the following way: Desintegrate $\mu_{u, x}$ according to Proposition 5.2 as $\mu_{u, x}(d v, d y)=\mu_{v}(d y) \rho(d v)$, where $\rho$ is the uniform distribution on $\left\{\theta_{t} u, t \in[0, T)\right\}$. Denote by $P_{t}=\left\{\varphi\left(\tau, \varphi(t, x, u), \theta_{t} u\right), \tau \in[0, T)\right\} \subset$ int $D \subset M$ the $T$-periodic trajectory of

$$
\dot{x}(t)=X_{0}(x(t))+\sum_{i=1}^{m} u_{i}(t+\cdot) X_{i}(x(t))=: Y(x(t))
$$

with initial value $\varphi(t, x, u), t \in[0, T)$. Define $\mu_{\theta_{t} u}$ to be the occupation measure on $P_{t}$; its density is given by $1 /|Y(z)|$, for $z \in P_{i}$. Then by construction $(u, x) \in \operatorname{supp} \mu_{u, x}$ and $\mu_{u, x}$ is ergodic, because for any $\phi$-invariant set $W \subset \mathscr{U} \times M$, we have either $\mu_{u, x} W=1$ (iff $(u, x) \in W$ ) or $\mu_{u, x} W=0$ (iff $(u, x) \notin W)$.

If $u \in \mathscr{U}$ is constant, or $\varphi(t, x, u)$ is constant, define the corresponding measures as the Dirac measures at this point.

Note that these constructions lead to product measures $\mu_{u, x}(d v, d y)=$ $\mu(d y) \rho(d v)$.

By Poincarés recurrence theorem we know that supp $\mu \subset \overline{\mathscr{R}} \subset \mathscr{U} \times M$ for all $\mu \in M_{\phi}$, where $\mathscr{R}$ denotes the set of $\phi$-recurrent points. But for general dynamical systems, it is not true that $\overline{\mathscr{R}}=\mathrm{cl}\left\{\bigcup_{\mu \in \mathscr{M}_{\phi}}\right.$ supp $\left.\mu\right\}$; see, e.g., Nemitsky and Stepanov (20), Section VI.3. For dynamical systems of the form (3.2) we obtain the following from Lemma 4.2 and Theorem 5.5(iii).

5.6. Corollary. Denote by $\mathscr{C}=\bigcup\left\{\mathscr{D}^{+}, D\right.$ is a control set of (3.1) $\}$ the union of all lifts of control sets as defined by (5.2). Then we have

$$
\overline{\mathscr{R}} \subset \overline{\mathscr{C}}, \quad \bigcup\left\{\operatorname{supp} \mu, \mu \in \mathscr{M}_{\phi}\right\} \subset \overline{\mathscr{C}}
$$

Furthermore, if the control sets have nonvoid interior, we obtain the following for the $M$-components by Lemma 4.2, and Theorem 5.5(iv).

5.7. Corollary. Assume that int $D \neq \varnothing$ for all control sets $D \subset M$ of (3.1). Then

$$
\begin{aligned}
\operatorname{cl} \bigcup\{D, D \text { is a control set }\} & =\pi_{M} \mathrm{cl} \bigcup\left\{\operatorname{supp} \mu_{u, x},(u, x) \in \Sigma_{\phi}^{s}\right\} \\
& =\pi_{M} \mathrm{cl} \bigcup\left\{\operatorname{supp} \mu, \mu \in M_{\phi}\right\}
\end{aligned}
$$


5.8. Remark. Let $\operatorname{dim} M=1$. The assumption (H) implies that all boundary points of control sets are steady states for some constant control $u \in \mathscr{U}$. Hence in this case

$$
\overline{\bigcup D}=\pi_{M} \mathscr{R}=\pi_{M} \bigcup_{(u, x) \in \Sigma_{\phi}^{s}} \operatorname{supp} \mu_{u, x}=\pi_{M} \bigcup_{\mu \in M_{\phi}} \operatorname{supp} \mu
$$

For $\operatorname{dim} M \geqslant 2$, however, there may be points in $\partial D$ that are in no support of any projected invariant measure.

5.9. Remark. If all $x \in M$ are in the support of some $\phi$-invariant measure, i.e., if $M=\pi_{M} \bigcup_{\mu \in M_{\phi}}$ supp $\mu$, then system (3.1) is not necessarily completely controllable on $M$; compare Example 4.3. But in this case, $M$ is one chain control set by Theorem 4.8 .

Of course, invariant measures of the dynamical system $(\mathscr{U} \times M, \phi)$ are in general not unique, if $U$ contains more than one point. In fact, the same $u \in \mathscr{U}$ can lead to different $\phi$-invariant measures [e.g., if (3.1) has several rest points corresponding to $u \in U]$, and the same $x \in M$ can be in the support (or can even coincide with the support) of several projected invariant measures. The question of uniqueness is more interesting, if one restricts the class of invariant measures. For stochastic systems, e.g., one assumes that a $\theta$-invariant (and ergodic) measure $\rho$ is given for $(\mathscr{U}, \theta)$, and one looks, e.g., for (unique) $\phi$-invariant measures corresponding to Markov processes, diffusion processes, etc., whose marginal on $\mathscr{U}$ is $\rho$. We refer to Crauel (12), Colonius and Kliemann (9), Kliemann (18), and Arnold and Kliemann (2) for some results in this direction.

\section{REFERENCES}

1. Arnold, L., and Kliemann, W. (1983). Qualitative theory of stochastic systems. In Bharucha-Reid (ed.), Probabilistic Analysis and Related Topics, Vol. III, Academic Press, New York, pp. 1-79.

2. Arnold, L., and Kliemann, W. (1987). On unique ergodicity for degenerate diffusions. Stochastics 21, 41-61.

3. Banks, J., Brooks, J., Cairns, G., Davis, G., and Stacey, P. (1992). On Devaney's definition of chaos. Am. Math. Month. 99, 332-334.

4. Bunke, H. (1972). Gewöhnliche Differentialgleichungen mit zufälligen Parametern, Akademie Verlag, Berlin.

5. Cohn, D. E. (1980). Measure Theory, Birkhäuser Verlag, Boston.

6. Colonius, F., and Kliemann, W. (1984). Infinite time optimal control and periodicity. Appl. Math. Optim. 20, 113-130.

7. Colonius, F., and Kliemann, W. (1993). Linear control semigroups acting on projective space. J. Dynam. Diff. Eq. 5, 495-528.

8. Colonius, F., and Kliemann, W. (1993). Limit behavior and genericity for nonlinear control systems. J. Diff. Eq. (in press). 
9. Colonius, F., and Kliemann, W. (1992). Remarks on ergodic theory of stochastic flows and control flows. In Pinsky, M., and Wihstutz, V. (eds.), Diffusion Processes and Related Problems in Analysis, Vol. II, Birkhäuser, pp. 203-240.

10. Conley, C. (1978). Isolated Invariant Sets and the Morse Index, CBMS Regional Conference Series, No. 38, American Mathematical Society, Providence.

11. Crauel, H. (1986). Lyapunov exponents and invariant measures of stochastic systems on manifolds. In Arnold, L., and Wihstutz, V. (eds.), Lyapunov Exponents, Lecture Notes in Mathematics, 1186, Springer-Verlag, Berlin, pp. 271-291.

12. Crauel, H. (1987). Random Dynamical Systems: Positivity of Lyapunov Exponents and Markov Systems, Dissertation, Universität Bremen, Bremen.

13. Devaney, R. L. (1986). An Introduction to Chaotic Dynamical Systems, Benjamin/ Cummings, Menlo Park, CA.

14. Dunford, N., and Schwartz, J. T. (1958). Linear Operators, Part I. General Theory, Wiley-Interscience, New York.

15. Engelking, R. (1977). General Topology, PWN-Polish Scientific, Warszawa.

16. Kaul, S. R. (1989). A semi-dynamical system associated with a general control system. Nonlin. Anal. 13, 1-5.

17. Gänssler, P., and Stute, W. (1977). Wahrscheinlichkeitstheorie, Springer-Verlag, Berlin.

18. Kliemann, W. (1987). Recurrence and invariant measures for degenerate diffusions. Ann. Prob. 15, 690-707.

19. Mañé, R. (1987). Ergodic Theory and Differentiable Dynamics, Springer-Verlag, New York.

20. Nemitsky, V. V., and Stepanov, V. V. (1960). Qualitative Theory of Differential Equations, Princeton University Press, Princeton, NJ.

21. Okikiolu, G. O. (1971). Aspects of the Theory of Bounded Integral Operators in $L^{p}$-Spaces, Academic Press, London-New York.

22. Roxin, E. (1965). Stability of general control systems. J. Diff. Eq. 1, 115-150.

23. Warga, J. (1972). Optimal Control of Ordinary and Functional Differential Equations, Academic Press, London-New York. 